\title{
MEG3 is a prognostic factor for CRC and promotes chemosensitivity by enhancing oxaliplatin-induced cell apoptosis
}

\author{
LIXIA LI, JIAN SHANG, YUPENG ZHANG, SHI LIU, YANAN PENG, ZHOU ZHOU, \\ HUAQING PAN, XIAOBING WANG, LIPNG CHEN and QIU ZHAO
}

Department of Gastroenterology/Hepatology, Zhongnan Hospital of Wuhan University, The Hubei Clinical Center and Key Laboratory of Intestinal and Colorectal Diseases, Wuhan, Hubei 430071, P.R. China

Received January 7, 2017; Accepted July 5, 2017

DOI: $10.3892 /$ or.2017.5828

\begin{abstract}
A major reason for the failure of advanced colorectal cancer (CRC) treatment is the occurrence of chemoresistance to oxaliplatin-based chemotherapy. Recently, studies have shown that long non-coding RNAs (lncRNAs) play an important role in drug resistance. Using HiSeq sequencing methods, we identified that lncRNAs show differential expression levels in oxaliplatin-resistant $(\mathrm{OxR})$ and non-resistant $\mathrm{CRC}$ patients. RT-qPCR was then performed in tissues and serum samples, and IncRNA MEG3 was verified to be downregulated in nonresponding patients and to have considerable discriminating potential to identify responding patients from non-responding patients. Moreover, decreased serum MEG3 expression was associated with poor chemoresponse and low survival rate in CRC patients receiving oxaliplatin treatment. Subsequently, OxR cell lines were established, and MEG3 was significantly downregulated in HT29 OxR and SW480 OxR cells. In addition, overexpression of MEG3 with pMEG3 reversed oxaliplatin resistance in both CRC cell lines. Flow cytometric apoptosis analysis indicated that MEG3 promoted CRC cell apoptosis. More importantly, MEG3 enhanced oxaliplatin-induced cell cytotoxicity in CRC. In conclusion, our integrated approach demonstrated that decreased expression of lncRNA MEG3 in CRC confers potent poor therapeutic efficacy, and that MEG3 promotes chemosensitivity by enhancing oxaliplatin-induced cell apoptosis. Thus, overexpression of MEG3 may be a future direction by which to develop a novel therapeutic strategy to overcome oxaliplatin resistance of CRC patients.
\end{abstract}

Correspondence to: Dr Lipng Chen or Dr Qiu Zhao, Department of Gastroenterology/Hepatology, Zhongnan Hospital of Wuhan University, Wuhan, Hubei 430071, P.R. China

E-mail: ningyaxiangslz@sina.com

E-mail: zhaoqiuhbwh@163.com

Key words: colorectal cancer, MEG3, oxaliplatin, chemoresistance, apoptosis

\section{Introduction}

Colorectal cancer (CRC) is a leading cause of cancerrelated deaths worldwide (1). It is the second and third most commonly diagnosed cancer in females and males, respectively, and more than 1.2 million patients are diagnosed with CRC every year $(2,3)$. Currently, oxaliplatin-based chemotherapy after surgical resection is one of the most widely used therapeutic strategies (4). However, a large proportion of patients receiving chemotherapy finally become metastatic and chemoresistant, and this has been a key barrier to the efficacy of CRC treatment (5). Hence, finding new therapeutic markers is warranted to evade drug resistance mechanisms, and possibly find a cure.

In recent years, a tremendous amount of effort has been devoted to understanding the mechanisms of chemoresistance and to elaborate the genes/pathways involved. Chemoresistance mechanisms are extraordinarily complex, including inefficient cellular drug uptake and accumulation (6), enhancement of DNA repair (7) and activation of the antioxidant glutathione system for detoxification $(8,9)$. One major resistance mechanism utilized by tumor cells is to resist drug-induced cell death through disruptions in apoptotic pathways (10). Thus, it is also essential to better understand the pathways related to chemoresistance in CRC and discover novel strategies to further improve the effectiveness of oxaliplatin.

Long non-coding RNAs (lncRNAs) are defined as transcripts $>200$ nucleotides in length and are transcribed, but are non-translated non-coding RNAs in the human genome (11). Recent studies have demonstrated that lncRNAs play important roles in carcinogenesis and cancer metastasis, and some lncRNAs function as oncogenes, tumor-suppressor genes or both, depending on the circumstance (12). However, aberrant IncRNA expression has been detected and identified as a promising biomarker for diagnosis and prognosis in breast and gastric cancer, and CRC (13-15). The discovery and study of lncRNAs are thus of major relevance to human biology and disease, as they represent an extensive, largely unexplored and functional component of the genome $(16,17)$. To date, lncRNA expression has not been extensively analyzed in CRC samples except in a few studies that have used microarrays from TCGA (18), and the genome-wide screening of relevant lncRNAs are essential for improving the prognosis of cancer 
patients. Moreover, the association of the expression of specific lncRNAs with drug resistance in CRC cells is not well known.

In the present study, we conducted high-throughput HiSeq sequencing followed by reverse transcription quantitative real-time PCR (RT-qPCR) assays to test the hypothesis that specific lncRNAs can be useful in predicting chemotherapeutic response with the hope that such findings may guide the therapeutic choice. Our data showed that MEG3 expression was significantly downregulated in primary tissues and serum samples from CRC patients showing resistance to oxaliplatinbased treatment. Moreover, the subsequent functional assay revealed that MEG3 reverses oxaliplatin resistance by promoting oxaliplatin-induced cell apoptosis.

\section{Materials and methods}

Patients and samples. A multiphase, case-control study was designed to identify lncRNAs as potential biomarkers for differentiating the chemoresponse to oxaliplatin therapy in CRC patients. Tumor response status was evaluated according to the Response Evaluation Criteria in Solid Tumors (RECIST) criteria and was assigned to patients with complete or partial response (CR and PR, respectively), and stable or progressive disease (SD and PD, respectively) in tumor measurements confirmed by repeat studies performed no less than 4 weeks after the criteria for response was first met. Briefly, 316 patients diagnosed with CRC, but without other diseases were recruited at Zhongnan Hospital of Wuhan University between January 2009 and February 2013. All participants were allocated to 3 phases. In the initial screening phase, tissue samples pooled from 8 patients showing response and 8 patients showing no response were subjected to HiSeq sequencing, to identify lncRNAs that were significantly differentially expressed. In the training phase, the candidate lncRNAs were tested with RT-qPCR in an independent cohort of primary tissues from 80 CRC patients responding to oxaliplatin treatment and 80 patients showing no response to treatment. In the validation phase, another independent group of $140 \mathrm{CRC}$ patients who provided serum samples were enrolled. Among these patients, there were 70 patients who showed response to oxaliplatin treatment while the other 70 patients showed no response.

All the patients were pathologically confirmed as presenting with CRC and the clinical tissue samples were collected before chemotherapy was started. They were classified according to the tumor-node-metastasis (TNM) classification. Overall survival (OS) was updated on February 1, 2012 and was defined as the time from inclusion to death for any reason. Recurrence-free survival (RFS) was defined as the time from inclusion to recurrence or metastatic progression.

Sample preparation. Fresh tumor tissues were immediately cut from the resected CRC tissues and kept at $-80^{\circ} \mathrm{C}$ until RNA extraction. Venous blood was collected and centrifuged at 4,000 rpm for $10 \mathrm{~min}$, within $2 \mathrm{~h}$. The supernatant fluids were then collected and further centrifuged at 12,000 rpm for $15 \mathrm{~min}$ to completely remove the cell debris. The whole process was strictly controlled to avoid hemolysis, and the supernatant serum was stored at $-80^{\circ} \mathrm{C}$, until analysis. Written informed consent was obtained from all patients according to local ethical regulations of the Ethics Committee of Zhongnan Hospital of Wuhan University.

HiSeq sequencing. Total tissue RNA was extracted by onestep extraction using a TRIzol reagent kit (Life Technologies, Carlsbad, CA, USA), and the purity and quantity of RNA were determined using UV spectrophotometry. cDNA library construction and sequencing were performed according to previously described methods (19). Briefly, after extraction of total RNA, ribosomal RNA was separated to isolate as much ncRNA as possible. RNA containing poly(A) was then removed. RNA fragments were broken into short fragments randomly. The first chain of cDNA was generated using RNA fragments as templates and 6-bp random primers. Second chain of the cDNA was synthesized according to the kit's instructions (Takara Bio Company, Dalian, China). After purification, end repair, base $\mathrm{A}$ and sequencing joint adding, the generated cDNA was fragmented using uracil-N-glycosylase (UNG). cDNA fragments were chosen according to size, then PCR amplification was performed to establish the complete sequencing cDNA library. lncRNAs were sequenced using the high-throughput, high-sensitivity HiSeq 2500 sequencing platform (Illumina Inc., San Diego, CA, USA). Sequencing results were analyzed and treated using Trim Galore software to dynamically remove joint sequence fragments and lowquality segments from the $3^{\prime}$ end. FastQC software was used for quality control of the pretreated data.

Cell culture. Human CRC cell lines HT29 and SW480 were obtained from the Type Culture Collection of the Chinese Academy of Sciences (Shanghai, China) in 2014. All CRC cell lines were maintained in RPMI-1640 medium (Thermo Fisher Scientific, Wilmington, DE, USA) containing 10\% fetal bovine serum (FBS) (Sigma-Aldrich, St. Louis, MO, USA), $100 \mathrm{U} / \mathrm{ml}$ penicillin and $100 \mathrm{~g} / \mathrm{ml}$ streptomycin (Life Technologies, Grand Island, NY, USA) at $37^{\circ} \mathrm{C}$ in $5 \% \mathrm{CO}_{2}$ and $95 \%$ air.

RNA extraction. Total RNA was isolated from primary CRC tissues and cells using TRIzol reagent (Invitrogen, Carlsbad, CA, USA). Serum RNA was isolated using acid phenol according to the manufacturer's instructions. The extracted total RNA was eluted in $20 \mu \mathrm{l}$ nuclease-free water and the RNA concentration was measured by NanoDrop 2000 (Thermo Fisher Scientific). The samples with A260/A280 nm ratios between 1.8 and 2.0 were used for further experiments.

Quantitative real-time PCR (RT-qPCR). For primary CRC tissues and cell lines, the cDNA was synthesized from $200 \mathrm{ng}$ extracted total RNA using the PrimeScript RT reagent kit (Takara Bio Company) and amplified by RT-qPCR using LightCycler 480 SYBR-Green I Master (Roche, Mannheim, Germany) and GAPDH was used as the control gene. For serum cell-free MEG3 detection, we used a previously established RT-qPCR-D method without RNA extraction (20). Briefly, the $2 \mathrm{X}$ preparation buffer was prepared, which contained $2.5 \%$ Tween-20 (EMD Chemicals, Gibbstown, NJ, USA), 50 mmol/1 Tris, and $1 \mathrm{mmol} / 1$ EDTA (both from Sigma-Aldrich). First, $5 \mu \mathrm{l}$ of serum was mixed with an equal volume of $2 \mathrm{X}$ preparation buffer. Subsequently, the above mixture was reverse 
transcribed (RT) in triplicates in a $20 \mu 1$ reaction volume. Finally, the RT product was 10 -fold diluted and centrifuged at $16,000 \mathrm{x}$ g for $5 \mathrm{~min}$, and $5 \mu \mathrm{l}$ supernatant solution was used as a cDNA template for qPCR. The reagents and reaction conditions were the same as those for RT-qPCR. The $2^{-\Delta \Delta C t}$ method was used to determine the relative quantification of gene expression levels. The primer sequences were as follows: MEG3 forward, 5'-CTGCCCATCTACACCTCACG-3' and reverse, 5'-CTCTCCGCCGTCTGCGCTAGGGGCT-3'; GAPDH forward, 5'-GCACCGTCAAGGCTGAGAAC-3' and reverse, ATGGTGGTGAAGACGCCAGT.

Development of oxaliplatin-resistant (OxR) cell lines. Oxaliplatin (Sanofi-Synthelabo, Hangzhou, China) was purchased from the pharmacy at Zhongnan Hospital of Wuhan University. HT29 and SW480 cells were exposed to an initial oxaliplatin concentration of $0.1 \mu \mathrm{mol} / 1$ in RPMI-1640 medium plus $10 \%$ FBS. The surviving population of cells was grown to $80 \%$ confluency and passaged twice over 9 days to ensure viability. The concentration of oxaliplatin that the surviving population was exposed to was then sequentially increased in the same manner to $0.5 \mu \mathrm{mol} / 1$ (15 days), $1.0 \mu \mathrm{mol} / 1$ (30 days), and finally to the clinically relevant plasma concentration of $2 \mu \mathrm{mol} / 1$. For all in vitro studies, OxR cells were used at no higher than 15 passages from creation and were maintained and exposed to $2 \mu \mathrm{mol} / 1$ oxaliplatin unless otherwise indicated.

Cell transfection. The MEG3 overexpression plasmid (pMEG3) and control vector (pVector) were purchased from Addgene (Cambridge, MA, USA). CRC cells were plated in 24 -well plates at $1 \times 10^{5} /$ well. Forty-eight hours after plating, $100 \mathrm{nM}$ of si-MEG3 or pMEG3 as well as the negative controls were transfected into the cells with Lipofectamine 2000 (Invitrogen) according to the manufacturer's instructions.

Cell viability assay. Cell viability was quantified using 3-(4,5-dimethylthiazol-2-yl)-2,5-diphenyl-tetrazolium bromide (MTT) (Sigma-Aldrich) assay. Briefly, $100 \mu \mathrm{l}$ of cells from the different groups was seeded onto a 96-well plate at a concentration of 5,000 cells/well and were incubated at $37^{\circ} \mathrm{C}$. At different time point, the optical density was measured at $450 \mathrm{~nm}$ using a microtiter plate reader, and the rate of cell survival was expressed as the absorbance. The concentration-dependent curves were generated based on the cell viability after the cells were cultured for $72 \mathrm{~h}$ at different concentrations of oxaliplatin. The results represent the mean of 3 replicates under the same conditions.

Cell apoptosis assay. Cells (1×10\%/well) were collected $48 \mathrm{~h}$ after transfection and were stained with Annexin V-FITC and propidium iodide (PI) according to the manufacturer's instructions (BD Biosciences, Erembodegem, Belgium). Apoptosis was assessed using flow cytometry (BD FACSCalibur; BD Biosciences).

Statistical analysis. Statistical analyses were performed using SPSS 19.0 software (SPSS, Inc., Chicago, IL, USA). Kolmogorov-Smirnov test was used to determine the normality of the distribution of data in each group. Data are presented as median (interquartile range). Difference in lncRNA levels among multiple groups in HiSeq sequencing was determined using Bonferroni adjustment method. Mann-Whitney U test or Kruskal-Wallis test was employed to compare differences of lncRNAs among clinical cohort groups. A log-rank test was used to analyze the statistical differences in survival as deduced from Kaplan-Meier curves. Cox proportional-hazard regression analysis was performed to calculate hazard ratio (HR) and 95\% confidence interval (CI) for each covariable. All differences were regarded as statistical significant when $\mathrm{P}<0.05$.

\section{Results}

Identification of candidate IncRNAs by high-throughput HiSeq sequencing. The HiSeq sequencing with 8 tissue samples pooled from CRC patients showing response and 6 from patients showing no response to oxaliplatin treatment was conducted. In total, 735 lncRNAs were identified with significant differential expression (fold-change, $\geq 2.0$ ). To identify the lncRNAs that are potential biomarkers, we concentrated on the top 60 most upregulated and 60 downregulated lncRNAs that were differentially expressed between CRC patients showing response or no response (Fig. 1). Starting from those lncRNAs with the greatest fold-change, we filtered appropriate candidate lncRNAs in descending order. Candidates should be plausible for primer designing, and only those having steady expression in tissue samples were selected. Finally, we chose 3 candidate lncRNAs from the upregulated group and 3 from the downregulated group as well (Table I). Another 4 lncRNAs were also tested by RT-qPCR since they were peviously shown to be dysregulated in CRC chemoresistance (21-24). Thus, 10 lncRNAs were selected as candidates for further testing via RT-qPCR.

MEG3 is downregulated in CRC patients showing response to oxaliplatin treatment by $R T-q P C R$. The expression of all 10 candidate IncRNAs was evaluated by RT-qPCR, using $80 \mathrm{CRC}$ tissues from patients showing response to oxaliplatin and 80 from patients showing no response. Among these, 4 lncRNAs (LOC286467, MEG3, LOC285194 and LincRNA-p21) were found to be significantly dysregulated in responding tissues compared with non-responding tissues (Table II). Subsequently, these 4 lncRNAs were further validated in an independent cohort of 140 serum samples from $70 \mathrm{CRC}$ patients showing response and 70 showing no response. Among the 4 candidate lncRNAs, only MEG3 was significantly desregulated with a markedly suppressed expression in non-responding patients compared to responding patients (Fig. 2). Thus, we focused on the role of MEG3 in CRC chemoresistance.

Decreased serum MEG3 expression predicts poor response to oxaliplatin treatment in CRC patients. Predictive biomarkers are better when blood-based, as blood is easily available and provides the chance to monitor cancer progression. Thus, receiver operator characteristic (ROC) curve analysis was firstly performed to investigate the potential value of serum MEG3 in distinguishing the chemotherapeutic responding and non-responding CRC patients. Our data showed that the area under the curve (AUC) was 0.784 , providing a diagnostic 


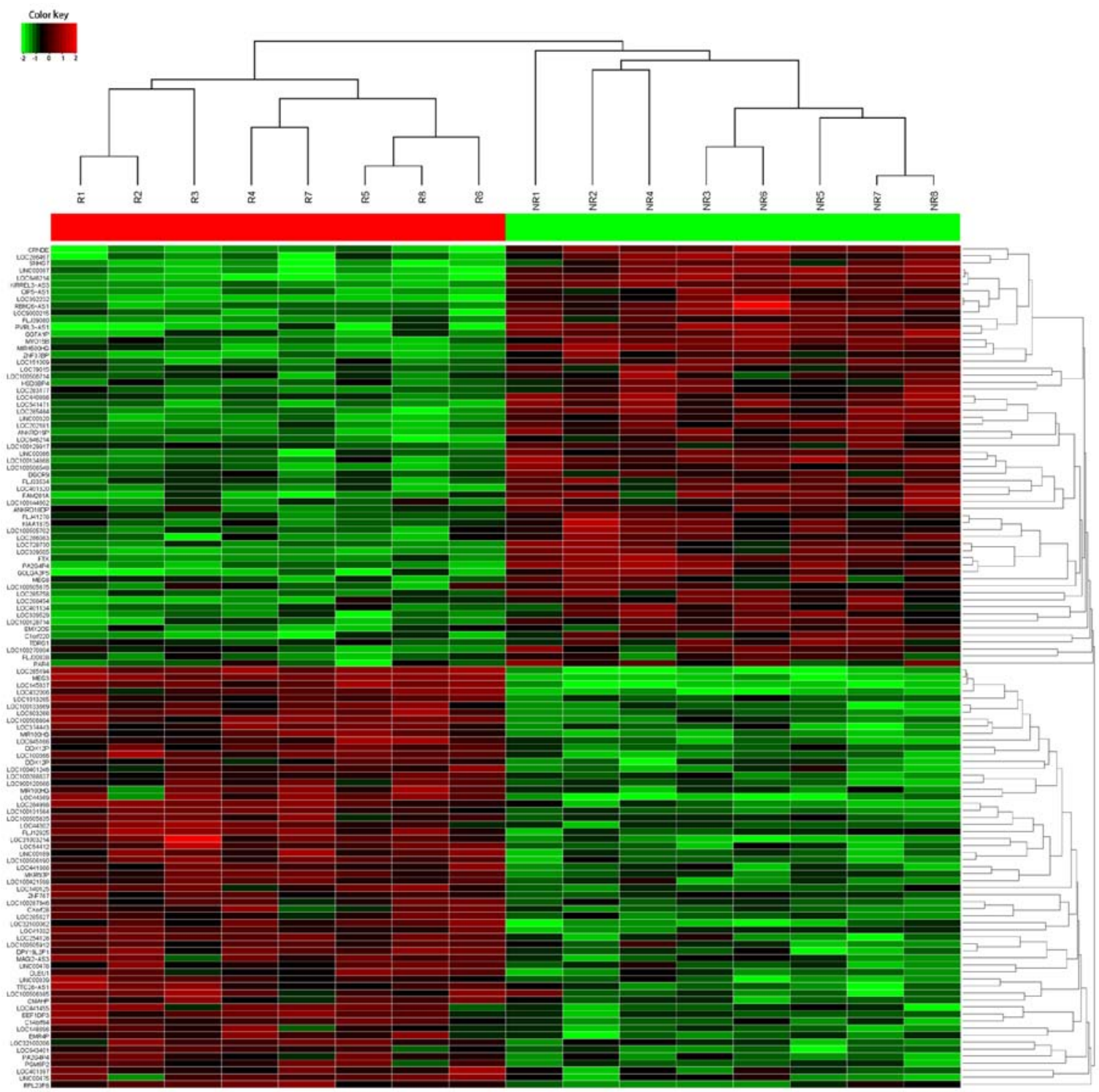

Figure 1. The heat map shows expression of the 120 lncRNAs most highly upregulated or downregulated in CRC responding compared with non-responding patients to oxaliplatin treatment. The top 60 lncRNAs that were upregulated or downregulated in the non-responding group are shown in the top and bottom halves, respectively. The heat map was generated with an $\mathrm{R}$ package using normalization across rows (tissues).

Table I. Candidate lncRNAs selected on a basis of the HiSeq analysis.

\begin{tabular}{llccc}
\hline Seqname & Location & Regulation (NR vs. R) & Fold-change & P-value \\
\hline LOC286467 & ChrXq26.2 & Up & 73.6341872 & 0.00006546 \\
CRNDE & Chr16q12.2 & Up & 42.6729041 & 0.00109347 \\
SNHG7 & Chr9q34.3 & Up & 27.8730264 & 0.00960371 \\
LOC145837 & Chr15q23 & Down & 48.8710538 & 0.00054087 \\
MEG3 & Chr14q32.2 & Down & 46.4837692 & 0.00079283 \\
LOC285194 & Chr3q13.13 & Down & 22.7845924 & 0.01947853 \\
\hline
\end{tabular}

NR, non-response; R, response.

sensitivity of $72.86 \%$ and a specificity of $61.43 \%$ (Fig. 3A) Under the stratification criteria (1.14) established by the ROC curve, patients were stratified into a high $(\mathrm{n}=62)$ and a low ( $\mathrm{n}=78)$ MEG3-expressing group. The proportion of patients that responded to oxaliplatin treatment was significantly higher in the high MEG3-expressing group than in 
Table II. Expression of 10 candidate lncRNAs in CRC patients showing response or non-response to oxaliplatin treatment [median (interquartile range)].

\begin{tabular}{lccr}
\hline IncRNA & Response & Non-response & P-value \\
\hline LOC286467 & $1.39(0.47-2.02)$ & $1.86(1.13-2.45)$ & $<0.05$ \\
CRNDE & $0.94(0.43-1.95)$ & $1.18(0.49-2.03)$ & 0.37 \\
SNHG7 & $1.14(0.33-2.51)$ & $1.57(0.52-3.08)$ & 0.24 \\
H19 & $0.87(0.35-1.89)$ & $1.27(0.44-1.97)$ & 0.06 \\
MALAT1 & $1.03(0.42-2.07)$ & $1.21(0.66-2.49)$ & 0.18 \\
LOC145837 & $0.99(0.38-2.15)$ & $0.77(0.23-1.85)$ & 0.09 \\
MEG3 & $1.32(0.40-2.31)$ & $0.74(0.35-1.62)$ & $<0.01$ \\
LOC285194 & $1.47(0.46-2.28)$ & $0.92(0.37-1.89)$ & $<0.01$ \\
LincRNA-p21 & $1.22(0.54-2.18)$ & $0.88(0.33-1.76)$ & $<0.05$ \\
SLC25A25-AS1 & $1.15(0.60-2.22)$ & $1.02(0.47-1.59)$ & 0.44 \\
\hline
\end{tabular}

lncRNA, long non-coding RNAs; CRC, colorectal cancer.
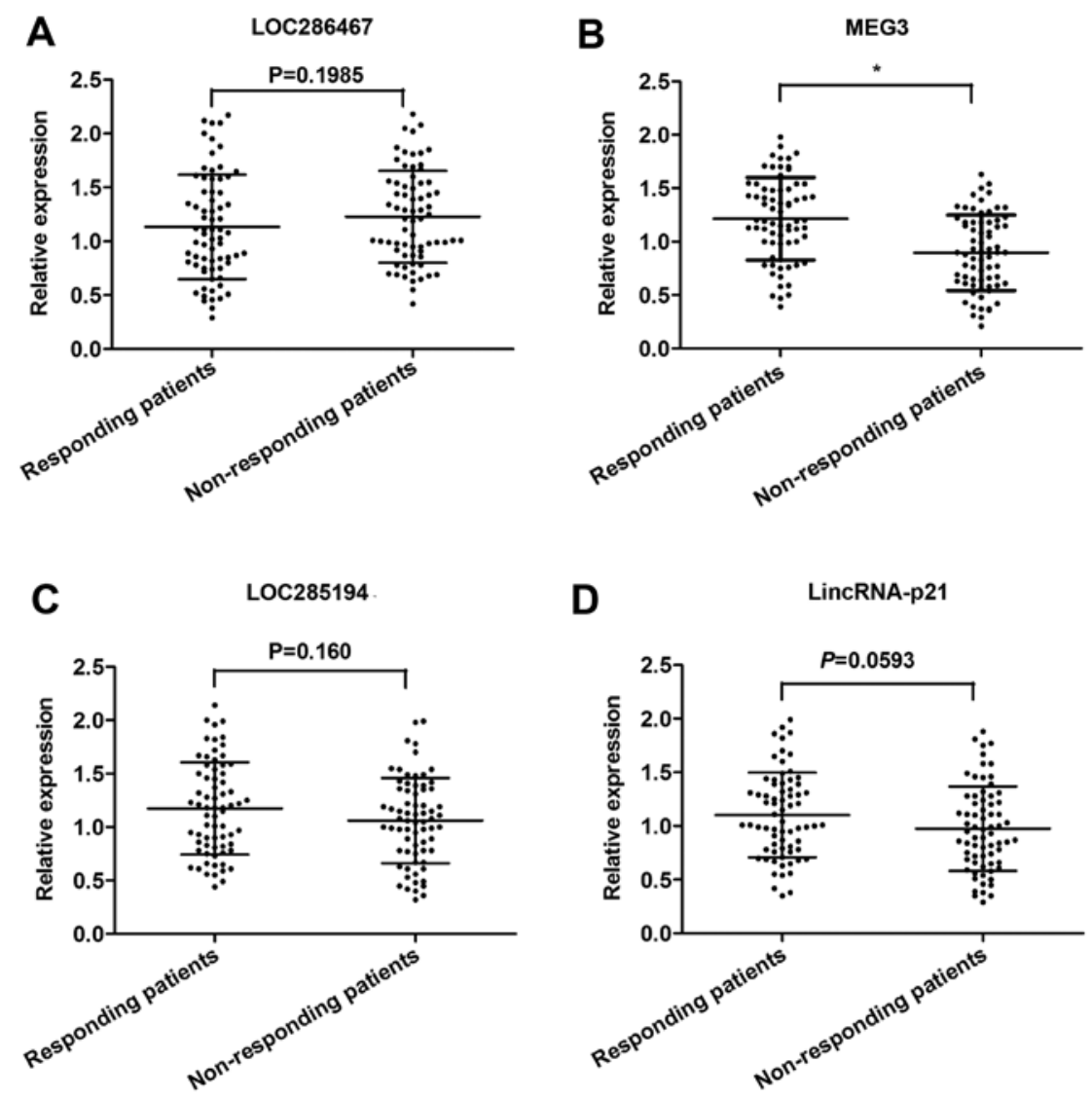

Figure 2. (A-D) Relative expression of 4 identified serum lncRNAs in CRC patients showing response ( $n=70)$ and non-response ( $n=70)$ to oxaliplatin therapy using RT-qPCR assay in a validation cohort. Error bars represent median \pm SD (standard deviation); ${ }^{*} \mathrm{P}<0.01$.

the low MEG3-expressing group ( $\mathrm{P}<0.01$; Fig. 3B). More importantly, Kaplan-Meier survival analysis was performed to further investigate the effect of serum MEG3 on oxaliplatin treatment for CRC patients. The results indicated that low MEG3 expression was associated with poor OS $(\mathrm{P}=0.007$; Fig. 3C) and RFS ( $\mathrm{P}=0.012$; Fig. 3D). Furthermore, we performed Cox regression univariate/mutivariate analyses to identify whether MEG3 or another clinical parameter was an independent indicator for OS of CRC patients who received oxaliplatin chemotherapy. The results indicated that the serum MEG3 expression level and distant metastasis maintained their significance as independent prognostic factors for OS of CRC patients receiving oxaliplatin treatment (Table III).

MEG3 is downregulated in OXR CRC cell lines. As a follow-up to our patient data that revealed a lower expression 
A

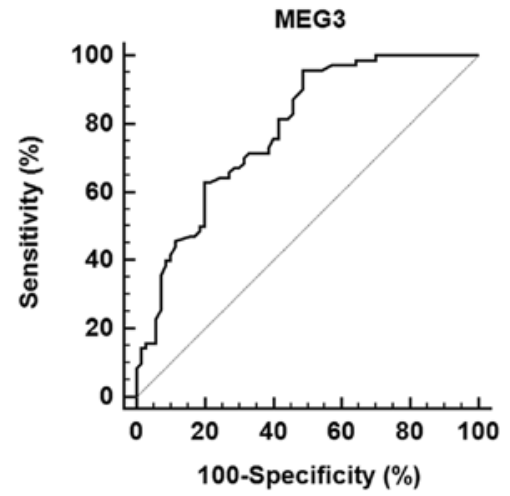

$\begin{array}{cccccc}\text { Criterion } & \text { Sensitivity (\%) } & \text { Specificity (\%) } & \text { AUC } & 95 \% \mathrm{Cl}(\%) \\ 1.14 & 72.86 & 61.43 & 0.784 & 0.707-0.849\end{array}$
B

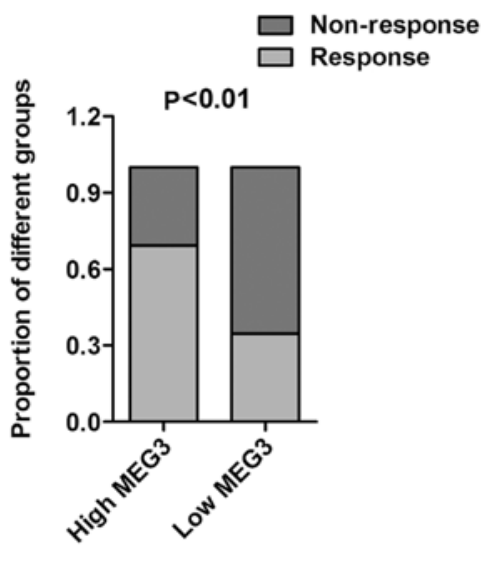

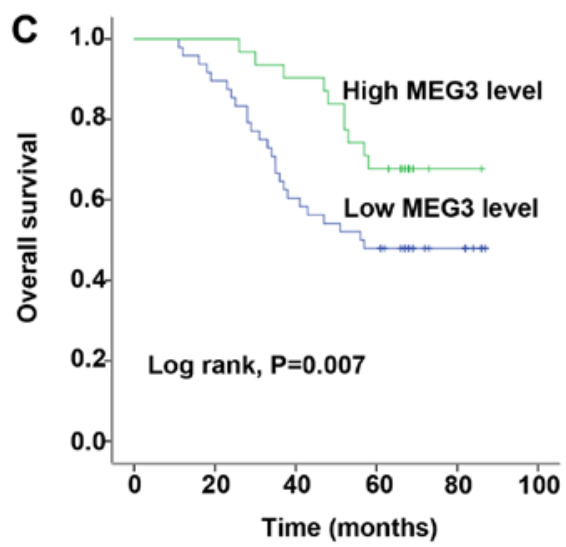

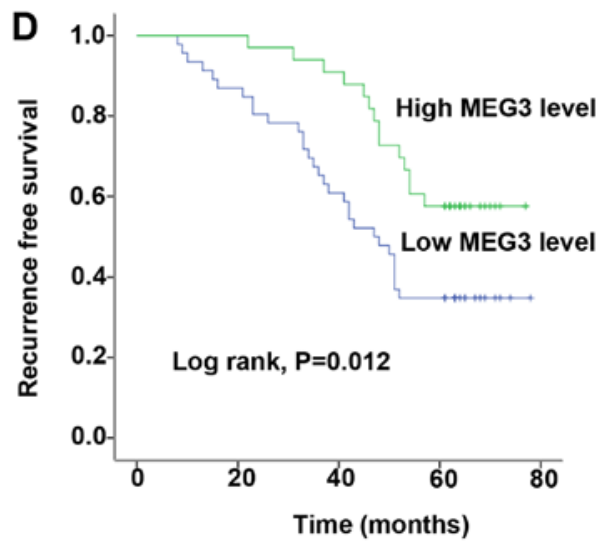

Figure 3. Decreased serum MEG3 expression is associated with poor response to oxaliplatin treatment in CRC patients. (A) ROC curves for differentiating the responding patients from non-responding patients of CRC using MEG3 expression in a validation cohort. (B) The proportion of patients that responded to oxaliplatin treatment was significantly higher in the high MEG3-expressing group than in the low-expressing group. (C and D) Kaplan-Meier curves for (C) OS and (D) RFS according to serum levels of MEG3 in CRC patients in the validation cohort. OS, overall survival; RFS, recurrence-free survival.

Table III. Univariate and multivariate Cox proportional hazards regression model analysis of factors for OS in patients with CRC in a validation cohort.

\begin{tabular}{|c|c|c|c|c|c|c|}
\hline \multirow[b]{2}{*}{ Characteristics } & \multicolumn{3}{|c|}{ Univariate analysis } & \multicolumn{3}{|c|}{ Multivariate analysis } \\
\hline & HR & $95 \% \mathrm{CI}$ & P-value & HR & $95 \% \mathrm{CI}$ & P-value \\
\hline Gender & 1.018 & $0.617-2.012$ & 0.639 & & & \\
\hline Age (years) & 1.533 & $0.741-2.882$ & 0.202 & & & \\
\hline Tumor size & 1.679 & $0.537-2.729$ & 0.418 & & & \\
\hline Differentiation & 1.885 & $1.029-3.352$ & 0.087 & & & \\
\hline Local invasion & 1.661 & $0.902-2.798$ & 0.152 & & & \\
\hline Distant metastasis & 2.771 & $1.580-3.998$ & 0.008 & 2.768 & $1.445-4.473$ & 0.007 \\
\hline TNM stage & 2.257 & $1.141-3.505$ & 0.043 & 2.285 & $1.027-3.555$ & 0.058 \\
\hline Serum MEG3 level & 1.353 & $0.321-2.107$ & 0.007 & 1.390 & $0.324-2.089$ & 0.007 \\
\hline
\end{tabular}

OS, overall survival; CRC, colorectal cancer; HR, hazard ratio; CI, confidence interval; TNM, tumor-node-metastasis.

of MEG3 in the OxR population, we further assessed the expression of MEG3 in the oxaliplatin-resistant CRC cell lines. HT29 and SW480 cell lines were constantly exposed to a high concentration of oxaliplatin $(2 \mu \mathrm{M})$ to establish the HT29 OxR (OxR) cell line and SW480 OxR cell line.
The established HT29-resistant cells were maintained and exposed to $2 \mu \mathrm{M}$ oxaliplatin unless otherwise indicated. As shown in Fig. 4A, both HT29 OxR and SW480 OxR cells showed elevated cell viability compared with the HT29 and SW480 parental cells when incubated with culture medium 

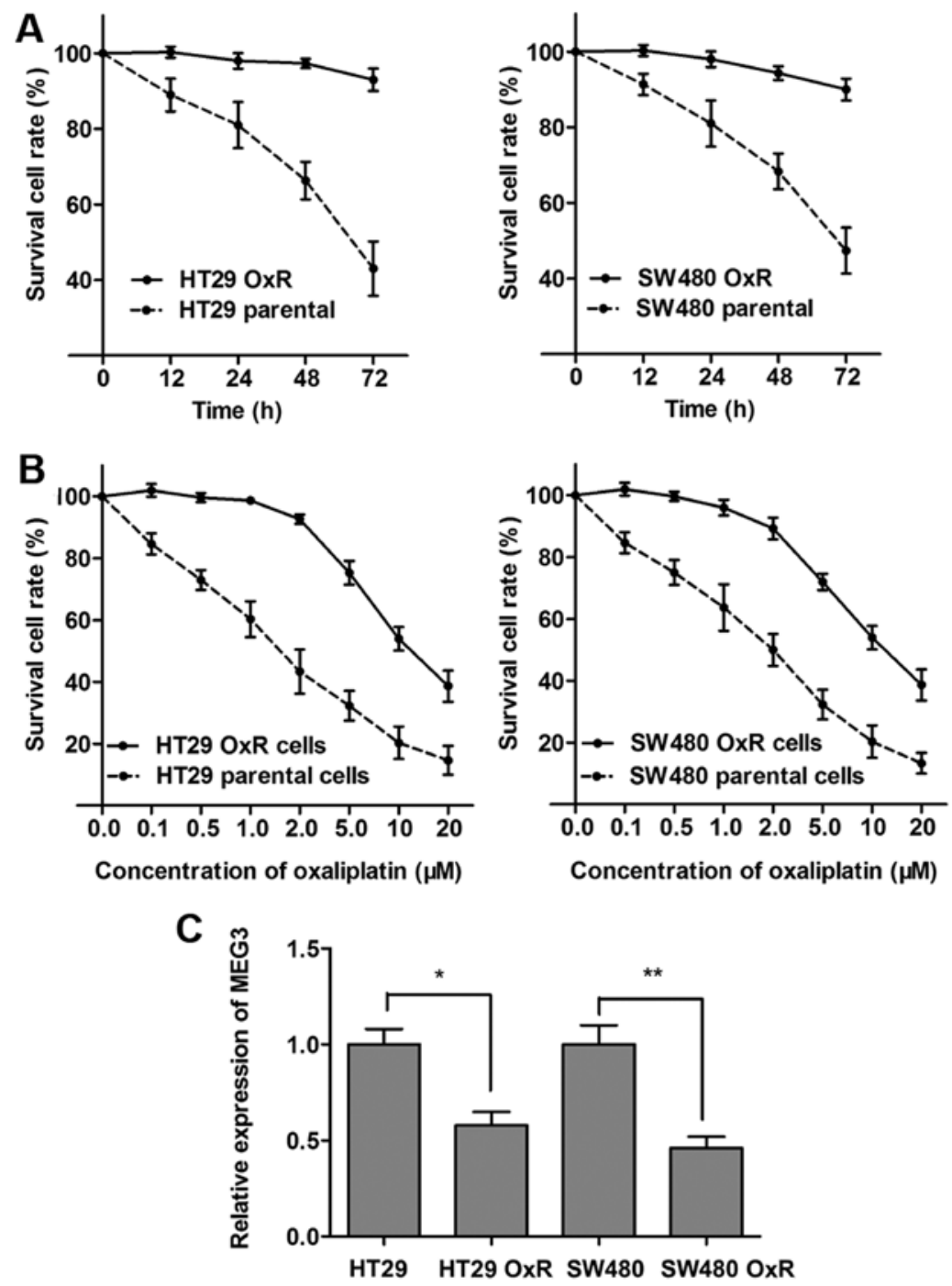

Figure 4. MEG3 is downregulated in oxaliplatin-resistant CRC cell lines. (A) Both HT29 OxR and SW480 OxR cell lines showed elevated cell viability compared to the HT29 and SW480 parental cells when incubated with culture medium containing $2 \mu \mathrm{M}$ concentration of oxaliplatin. (B) The concentrationeffect curve indicated that the $\mathrm{IC}_{50}$ values of oxaliplatin for $\mathrm{HT} 29 \mathrm{OxR}(11.6 \mu \mathrm{M})$ and SW480 OxR cells $(10.3 \mu \mathrm{M})$ were significant higher than that for the HT29 $(1.5 \mu \mathrm{M})$ and SW480 parental cells $(1.9 \mu \mathrm{M})$. (C) The expression of MEG3 in HT29 OxR and SW480 OxR cells was significantly lower than that in the HT29 and SW480 parental cells, respectively. Error bars represent SD. ${ }^{*} \mathrm{P}<0.05 ;{ }^{* *} \mathrm{P}<0.01$.

containing $2 \mu \mathrm{M}$ concentration of oxaliplatin. In contrast, the concentration-effect curve indicated that the $\mathrm{IC}_{50}$ value of oxaliplatin for HT29 OxR cells was $11.6 \mu \mathrm{M}$, while the $\mathrm{IC}_{50}$ value of oxaliplatin for the HT29 parental cells was $1.5 \mu \mathrm{M}$, which means that the HT29 OxR cells exhibited a 7.7 times higher ability of oxaliplatin resistance than the HT29 cells. Similarly, the SW480 OxR cells exhibited a 5.4 times higher ability of oxaliplatin resistance than the SW480 parental cells (10.3/1.9 $\mu \mathrm{M}$; Fig. 4B). After the OxR CRC sub-lines were established, we determined the MEG3 expression level, and found that MEG3 was significantly downregulated in the HT29 OxR and SW480 OxR cells compared with the parental HT29 and SW480 cells, respectively (Fig. 4C).

Overexpression of MEG3 partially reverses the chemoresistance status of OxR cells. After having validating the disruption of MEG3 in OxR cells, we further investigated whether MEG3 plays a role during the formation of oxaliplatin resistance in CRC cells. MEG3 overexpression plasmid (pMEG3) was trans- fected into HT29 OxR and SW480 OxR cells, and RT-qPCR assay showed that the MEG3 expression level was significantly increased in cells transfected with pMEG3 (Fig. 5A). Moreover, the survival cell rate was significantly impaired when MEG3 was overexpressed in the HT29 OxR and SW480 OxR cells incubated with $2 \mu \mathrm{M}$ concentration of oxaliplatin (Fig. 5B and C). This suggests that MEG3 overexpression partially reverses the oxaliplatin resistance in CRC.

MEG3 promotes oxaliplatin-induced apoptosis in CRC cells. Previous studies have demonstrated that MEG3 may have an effect on cell apoptosis during cancer angiogenesis (25). Thus, flow cytometric apoptosis analysis was performed to explore whether MEG3 reversed oxaliplatin resistance through promotion of cell apoptosis. Our results showed that pMEG3 significantly enhanced cell apoptosis in the HT29 and SW480 cells (Fig. 6A). To directly validate that MEG3 enhanced oxaliplatin-induced cell apoptosis in CRC, we treated HT29 and SW480 cells with a concentration gradient 

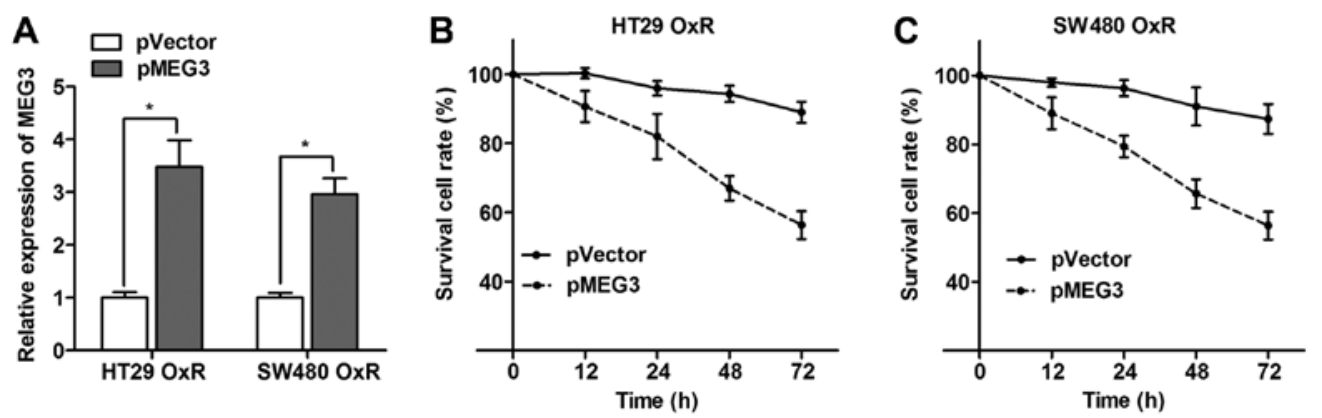

Figure 5. Overexpression of MEG3 reverses the chemoresistance of oxaliplatin-resistant CRC cells. (A) MEG3 expression level was significantly increased after transfection of pMEG3 in both HT29 OxR and SW480 OxR cells. (B and C) Cell viability was significantly impaired when MEG3 was overexpressed in the HT29 OxR (B) and SW480 OxR cells (C) compared with the negative control vector. Error bars represent SD. "P<0.001.
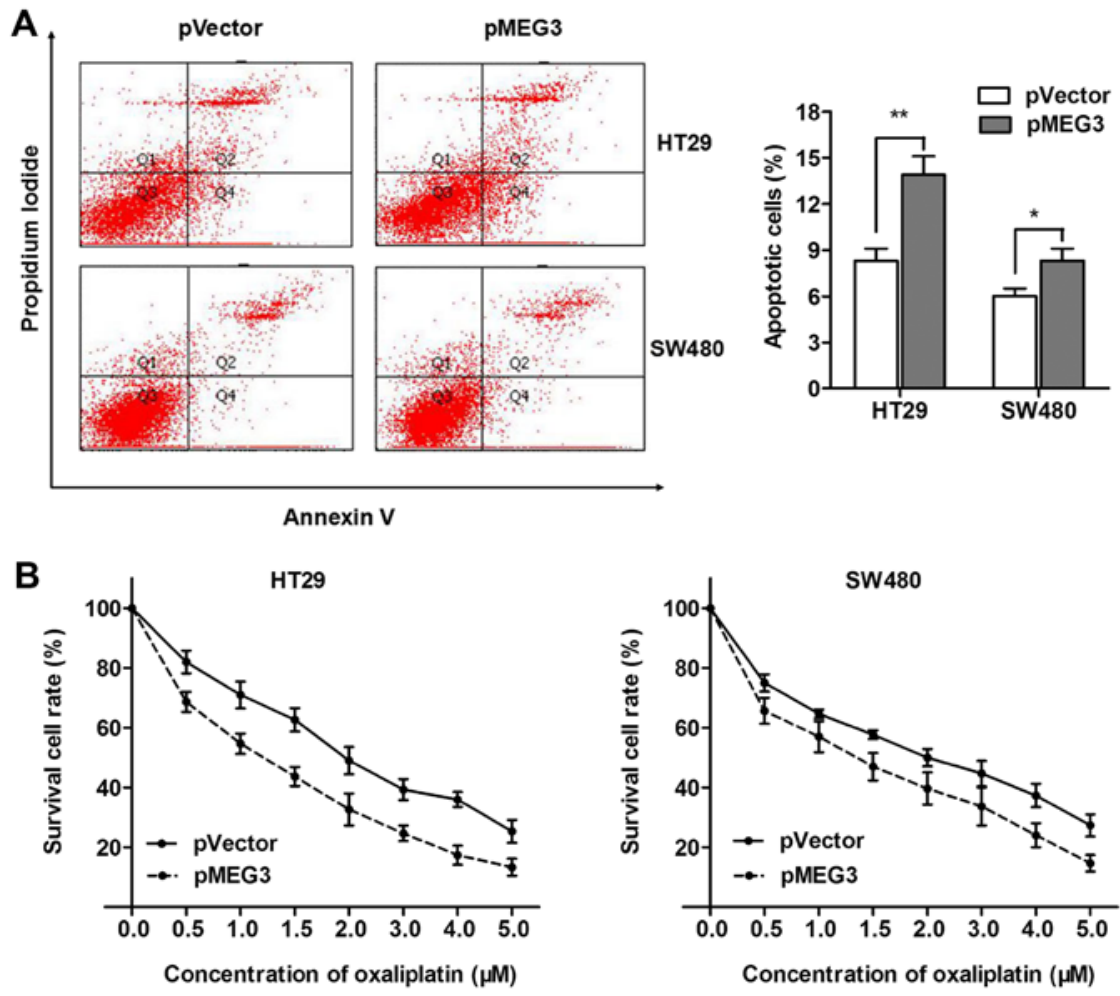

Figure 6. MEG3 promotes oxaliplatin-induced apoptosis in CRC cells. (A) Overexpression of MEG3 with pMEG3 significantly promoted apoptosis in both HT29 and SW480 cell lines. (B) The dose-effect curve showed that pMEG3 transfection was followed by increased cell death compared with the pVector control in the HT29 and SW480 cells. Error bars represent SD. ${ }^{*} \mathrm{P}<0.05 ;{ }^{* *} \mathrm{P}<0.01$.

of 0-5 $\mu \mathrm{M}$ oxaliplatin after being transfected with pMEG3 or the negative control. The dose-effect curve showed that pMEG3 transfection was followed by significantly increased cell apoptosis compared with the pVector control (Fig. 6B). However, the $\mathrm{IC}_{50}$ values for oxaliplatin were 1.8 and $2 \mu \mathrm{M}$ in the non-transfected HT29 and SW480 cells, and were markedly decreased to 1.2 and 1.4 after transfection with pMEG3, respectively. To conclude, MEG3 enhanced the chemosensitivity of oxaliplatin by promoting oxaliplatin-induced apoptosis in CRC cells.

\section{Discussion}

Despite recent chemotherapeutic regimens that have significantly increased the survival of patients with metastatic disease, invariably, nearly all CRC patients finally become chemoresistant accompanied by distant metastasis (5). Identification of new therapeutic markers and better understanding of the pathways related to chemoresistance are essential to improving the prognosis of CRC patients. In the present study, high-throughput HiSeq sequencing was firstly employed to provide basic information concerning the lncRNAs significantly dysregulated in CRC tissues. Candidate lncRNAs were selected and then evaluated by RT-qPCR in tissues and serum samples to validate their consistent pattern of dysregulation in these clinical materials. MEG3 was finally identified to show considerable discriminating potential to identify responding patients from non-responding patients with high AUC value. Serum MEG3 expression was associated with chemoresponse and predicted the prognosis of CRC 
patients receiving oxaliplatin treatment. More importantly, we demonstrated the molecular mechanism by which MEG3 exerted its function in oxaliplatin chemoresistance in CRC cells. We found that MEG3 promoted chemosensitivity by enhancing oxaliplatin-induced cell cytotoxicity in CRC cells.

In recent years, surgical resection with subsequent firstline chemotherapy regimens are common clinical therapeutic strategies. A major challenge, however, is that only approximately half of the patients obtain an objective response to the regimens, and that partial cross-resistance exists between different drugs (26). For this reason, it is of utmost importance to identify molecular bio-markers that have effective diagnostic and prognostic meaning. In the present study, we systematically investigated the expression of specific lncRNAs using a 3-phase study. IncRNAs dysregulated in both tissue and serum with a consistent pattern could effectively represent the lncRNA expression alteration in CRC and simultaneously satisfy the demand of noninvasive biomarkers. This stringent analysis led to identification of only one significantly altered lncRNA, MEG3.

MEG3 is located on chromosome $14 q 32$ and is widely expressed in many types of normal tissues $(27,28)$. MEG3 was first identified as the ortholog of gene traplocus 2 (Gtl2) in mice (29). It belongs to the DLK1-MEG3 imprinting locus which contains some maternally and paternally imprinted genes (30). Accumulating studies have demonstrated that MEG3 expression is downregulated in various types of cancers (27). Thus, MEG3 is a tumor-suppressor and overexpression of MEG3 could inhibit tumor cell proliferation and promote tumor cell apoptosis in different cancers $(13,31-33)$. In our research, we confirmed the downregulation of MEG3 and found that serum MEG3 expression considerably distinguished responding patients from non-responding ones with high diagnostic efficiency. However, the proportion of patients that responded to oxaliplatin treatment was significantly higher in the high MEG3-expressing group than in the low MEG3-expressing group. Most importantly, low serum MEG3 expression was associated with poor OS and RFS in patients receiving oxaliplatin treatment. Collectively, we revealed that MEG3 is a lncRNA lowly expressed in non-responding CRC patients and closely correlated to the chemoresponse to oxaliplatin treatments.

Taking one step further, we aimed to verify the underlying regulatory mechanism that could account for these findings. By establishing two oxaliplatin-resistant cell lines, we revealed that the MEG3 expression level was significantly suppressed in oxaliplatin-resistant cells compared with that noted in the parental cells. However, MEG3 overexpression promoted while MEG3 knockdown inhibited CRC cell apoptosis. More importantly, enhanced MEG3 expression significantly promoted oxaliplatin-induced cell cytotoxicity in the CRC cell lines. This indicates that MEG3 overexpression can reverse oxaliplatin resistance in CRC cells; thus, suppression of expression of MEG3 may be an important contributor to oxaliplatin resistance of CRC. Our results are partly consistent with previous studies. Yin et al found that MEG3 suppressed cell proliferation, caused cell cycle arrest and promoted cell apoptosis in CRC (13). Chak et al demonstrated that MEG3 is also downregulated in nasopharyngeal carcinoma (NPC) and predicted better survival by promoting
NPC cell apoptosis (33). Luo et al also found that MEG3 inhibited cell proliferation and induced apoptosis in prostate cancer (34). Thus, our data demonstrated that MEG3 promoted oxaliplatin-induced cell apoptosis in CRC, which may explain why MEG3 is downregulated in non-responding CRC patients and oxaliplatin-resistant cells.

In conclusion, our integrated approach demonstrated that MEG3 expression is downregulated in CRC patients who are resistant to oxaliplatin treatment, and is closely associated with the chemoresponse status to oxaliplatin treatment. We also revealed that MEG3 promoted the chemosensitivity to oxaliplatin of CRC by enhancing oxaliplatin-induced cell cytotoxicity. These findings indicate that downregulation of MEG3 confers a potent poor therapeutic efficacy. Thus, MEG3 may be a potential prognostic marker and therapeutic target in CRC patients.

\section{Acknowledgements}

The authors would like to thank Professor Bing Xia for his great contribution to their study. The present study was supported by research grants from the National Natural Science Foundation of China (no. 81270467).

\section{References}

1. Torre LA, Bray F, Siegel RL, Ferlay J, Lortet-Tieulent J and Jemal A: Global cancer statistics, 2012. CA Cancer J Clin 65: 87-108, 2015.

2. Li PL, Zhang X, Wang LL, Du LT, Yang YM, Li J and Wang CX: MicroRNA-218 is a prognostic indicator in colorectal cancer and enhances 5-fluorouracil-induced apoptosis by targeting BIRC5. Carcinogenesis 36: 1484-1493, 2015.

3. Han D, Wang M, Ma N, Xu Y, Jiang Y and Gao X: Long non-coding RNAs: Novel players in colorectal cancer. Cancer Lett 361: 13-21, 2015.

4. Yang AD, Fan F, Camp ER, van Buren G, Liu W, Somcio R, Gray MJ, Cheng H, Hoff PM and Ellis LM: Chronic oxaliplatin resistance induces epithelial-to-mesenchymal transition in colorectal cancer cell lines. Clin Cancer Res 12: 4147-4153, 2006.

5. Goldberg RM, Sargent DJ, Morton RF, Fuchs CS, Ramanathan RK, Williamson SK, Findlay BP, Pitot HC and Alberts SR: A randomized controlled trial of fluorouracil plus leucovorin, irinotecan, and oxaliplatin combinations in patients with previously untreated metastatic colorectal cancer. J Clin Oncol 22: 23-30, 2004.

6. Hector S, Bolanowska-Higdon W, Zdanowicz J, Hitt S and Pendyala L: In vitro studies on the mechanisms of oxaliplatin resistance. Cancer Chemother Pharmacol 48: 398-406, 2001.

7. Ahmad S: Platinum-DNA interactions and subsequent cellular processes controlling sensitivity to anticancer platinum complexes. Chem Biodivers 7: 543-566, 2010.

8. Sau A, Pellizzari Tregno F, Valentino F, Federici $G$ and Caccuri AM: Glutathione transferases and development of new principles to overcome drug resistance. Arch Biochem Biophys 500: 116-122, 2010.

9. Landriscina M, Maddalena F, Laudiero G and Esposito F: Adaptation to oxidative stress, chemoresistance, and cell survival. Antioxid Redox Signal 11: 2701-2716, 2009.

10. Gottesman MM, Fojo T and Bates SE: Multidrug resistance in cancer: Role of ATP-dependent transporters. Nat Rev Cancer 2: 48-58, 2002.

11. Kapranov P, Cheng J, Dike S, Nix DA, Duttagupta R, Willingham AT, Stadler PF, Hertel J, Hackermüller J, Hofacker IL, et al: RNA maps reveal new RNA classes and a possible function for pervasive transcription. Science 316: 1484-1488, 2007.

12. Fan Y, Shen B, Tan M, Mu X, Qin Y, Zhang F and Liu Y: TGF- $\beta$-induced upregulation of malat 1 promotes bladder cancer metastasis by associating with suz12. Clin Cancer Res 20: $1531-1541,2014$ 
13. Yin DD, Liu ZJ, Zhang E, Kong R, Zhang ZH and Guo RH: Decreased expression of long non-coding RNA MEG3 affects cell proliferation and predicts a poor prognosis in patients with colorectal cancer. Tumour Biol 36: 4851-4859, 2015.

14. Lai Y,Xu P,Li Q, Ren D, Wang J, Xu K and Gao W: Downregulation of long non-coding RNA ZMAT1 transcript variant 2 predicts a poor prognosis in patients with gastric cancer. Int J Clin Exp Pathol 8: 5556-5562, 2015.

15. Yang F, Liu YH, Dong SY, Ma RM, Bhandari A, Zhang XH and Wang OC: A novel long non-coding RNA FGF14-AS2 is correlated with progression and prognosis in breast cancer. Biochem Biophys Res Commun 470: 479-483, 2016.

16. Ponting CP, Oliver PL and Reik W: Evolution and functions of long non-coding RNAs. Cell 136: 629-641, 2009.

17. Mattick JS: The genetic signatures of non-coding RNAs. PLoS Genet 5: e1000459, 2009.

18. Wang R, Du L, Yang X, Jiang X, Duan W, Yan S, Xie Y, Zhu Y, Wang Q, Wang L, et al: Identification of long non-coding RNAs as potential novel diagnosis and prognosis biomarkers in colorectal cancer. J Cancer Res Clin Oncol 142: 2291-2301, 2016.

19. Iyer MK, Niknafs YS, Malik R, Singhal U, Sahu A, Hosono Y, Barrette TR, Prensner JR, Evans JR, Zhao S, et al: The landscape of long non-coding RNAs in the human transcriptome. Nat Genet 47: 199-208, 2015.

20. Zhang X, Yang X, Zhang Y, Liu X, Zheng G, Yang Y, Wang L, Du L and Wang C: Direct serum assay for cell-free bmi-1 mRNA and its potential diagnostic and prognostic value for colorectal cancer. Clin Cancer Res 21: 1225-1233, 2015.

21. Li Y, Huang S, Li Y, Zhang W, He K, Zhao M, Lin H, Li D, Zhang H, Zheng Z, et al: Decreased expression of LncRNA SLC25A25-AS1 promotes proliferation, chemoresistance, and EMT in colorectal cancer cells. Tumour Biol 37: 14205-14215 2016.

22. Li Q, Dai Y, Wang F and Hou S: Differentially expressed long non-coding RNAs and the prognostic potential in colorectal cancer. Neoplasma 63: 977-983, 2016.

23. Wu KF, Liang WC, Feng L, Pang JX, Waye MM, Zhang JF and $\mathrm{Fu}$ WM: H19 mediates methotrexate resistance in colorectal cancer through activating Wnt/ $\beta$-catenin pathway. Exp Cell Res 350: 312-317, 2017.
24. Wang J, Lei ZJ, Guo Y, Wang T, Qin ZY, Xiao HL, Fan LL, Chen DF, Bian XW, Liu J, et al: miRNA-regulated delivery of lincRNA-p21 suppresses $\beta$-catenin signaling and tumorigenicity of colorectal cancer stem cells. Oncotarget 6: 37852-37870, 2015.

25. Kumar MM and Goyal R: LncRNA as a therapeutic target for angiogenesis. Curr Top Med Chem 17: 1750-1757, 2017. doi: 10.2 174/1568026617666161116144744.

26. Pfeiffer P, Qvortrup C and Eriksen JG: Current role of antibody therapy in patients with metastatic colorectal cancer. Oncogene 26: 3661-3678, 2007.

27. Benetatos L, Vartholomatos G and Hatzimichael E: MEG3 imprinted gene contribution in tumorigenesis. Int J Cancer 129: 773-779, 2011.

28. Zhou Y, Zhangxand Klibanski A: MEG3 non-coding RNA: A tumor suppressor. J Mol Endocrinol 48: R45-R53, 2012.

29. Schuster-Gossler K, Bilinski P, Sado T, Ferguson-Smith A and Gossler A: The mouse Gtl2 gene is differentially expressed during embryonic development, encodes multiple alternatively spliced transcripts, and may act as an RNA. Dev Dyn 212: 214-228, 1998.

30. Miyoshi N, Wagatsuma H, Wakana S, Shiroishi T, Nomura M, Aisaka K, Kohda T, Surani MA, Kaneko-Ishino T, Ishino F: Identification of an imprinted gene, Meg3/Gtl2 and its human homologue $M E G 3$, first mapped on mouse distal chromosome 12 and human chromosome 14q. Genes Cells 5: 211-220, 2000.

31. Balik V, Srovnal J, Sulla I, Kalita O, Foltanova T, Vaverka M, Hrabalek L and Hajduch M: MEG3: A novel long non-coding potentially tumour-suppressing RNA in meningiomas. J Neurooncol 112: 1-8, 2013.

32. Wang P, Ren Z and Sun P: Overexpression of the long non-coding RNA MEG3 impairs in vitro glioma cell proliferation. J Cell Biochem 113: 1868-1874, 2012.

33. Chak WP, Lung RW, Tong JH, Chan SY, Lun SW, Tsao SW, Lo KW and To KF: Downregulation of long non-coding RNA MEG3 in nasopharyngeal carcinoma. Mol Carcinog 56: 1041-1054, 2017.

34. Luo G, Wang M, Wu X, Tao D, Xiao X, Wang L, Min F, Zeng F and Jiang G: Long non-coding RNA MEG3 inhibits cell proliferation and induces apoptosis in prostate cancer. Cell Physiol Biochem 37: 2209-2220, 2015. 